\title{
Propuesta de medición para toma de decisiones sobre el consumo de energía eléctrica
}

\section{Proposal for measurement decisions on electrical energy consumption}

Recibido: 10/07/2013

Revisado: 02/09/2013

Aceptado: 06/11/2013

\author{
Claudia García-Landa y María Montero \\ Universidad Nacional Autónoma de México, México
}

Contacto con autores:

Claudia García - Landa. cgarlan1@yahoo.com.mx.

María Montero.montero@.unam.mx

\section{Resumen}

This paper aims to document the psychometric characteristics of an instrument to assess decision-making with regard to power consumption (TDCEE) by means of narrative vignettes. The instrument was developed based on the assumption that decision-making in descriptive models is a dual rational and intuitive process. Based on the results of factorial analysis, two dimensions were identified (TDI and TDR) with moderate alphas and low correlation coefficient between them. We conclude that this scale meets acceptable psychometric criteria to be applied in the Mexican population.

Key words: decision-making, power, vignettes, measurement, narratives

En las grandes metrópolis como México, D.F., se requiere de una gran cantidad de bienes y servicios para satisfacer las necesidades de alimento, educación, vivienda, transporte, entretenimiento y salud de los ciudadanos. La satisfacción de estas necesidades provoca serios problemas ambientales como sobre explotación de mantos acuíferos, tala de árboles, invasión y destrucción de áreas verdes, generación de basura y emisiones de contaminantes al aire, entre otros
(Comisión Ambiental Metropolitana, 2010; PNUMA, 2013). En respuesta a estos problemas, el gobierno de la Ciudad de México ha empezado a incluir en su discurso político, mediante acuerdos como el Pacto de la Ciudad de México (Natyelly, 2010), medidas que involucren a los ciudadanos tal como su participación en la lucha contra el cambio climático a través de hacer modificaciones en sus estilos de vida. Lo anterior, conlleva a que los ciudadanos 
hagan cambios en su vida cotidiana para un consumo responsable, en este caso, de energía eléctrica. No obstante, los ciudadanos como consumidores no sólo buscan satisfacer sus necesidades de confort sino también obtener mayor placer a través del consumo excesivo de bienes y servicios (Mirosa, Lawson \& Gnoth, 2011; Shrogren, 2012). Desatender el problema de decidir por un consumo responsable de energía eléctrica en la vivienda mexicana podría llevar, por una parte, a tomar medidas de racionalización del servicio como sucedió en 2001 en Estados Unidos, Brasil, Suecia y Nueva Zelanda y en 2003 en Europa y Canadá (Organization for Economic Co-operation and Development, 2005) afectando principalmente la actividad económica del país. Por otra parte, esta problemática puede continuar mermando la economía familiar y elevando el número de emisiones de gases contaminantes al aire, ya que el consumo de energía eléctrica en la Ciudad de México representa un gasto del $20 \%$ del ingreso mensual familiar promedio (Instituto Nacional de Estadística, Geografía e Informática INEGI, 2012) y causa el $80 \%$ de las emisiones al aire de gases contaminantes por la producción y generación de energía eléctrica en plantas termoeléctricas (Comisión Federal de Electricidad CFE, 2010). De ahí la importancia de las medidas implementadas por el gobierno de la Ciudad de México y de las decisiones que tome el ciudadano sobre si optar por el consumo responsable de la energía eléctrica.

Investigadores de diversas áreas como la económica, educativa, mercadológica y psicológica, entre otras, han estudiado la toma de decisiones sobre el consumo (Brohmann et al., 2009). En la última década se ha incrementado el interés por su estudio apareciendo a nivel internacional diversas revistas (e.g. Judgement Under Risk, Judgement and Decision Making, Behavioral Decision Making, Journal of Consumer Research, Journal of Consumer Marketing, Journal of Consumer Psychology y Organizational Behavior and Human Decision Processes).

La toma de decisiones ha sido analizada desde dos tipos de modelos: los normativos y los descriptivos. Los modelos normativos tienen como objetivo explicar el resultado de la toma de decisiones basados en la elección final realizada por la persona; ejemplo de ello son los modelos de maximización de utilidad y utilidad esperada (ver Vanderberg, Carrico \& Schultz, 2011 para obtener más detalles). Los modelos descriptivos destacan la importancia de estudiar el proceso de las decisiones más que el resultado, es decir, lo que la persona hace al tomar decisiones (Heinzle, 2010). Dentro de éstos modelos se encuentran los de racionalidad limitada, estructura y dependencia de la referencia, irracionalidad y heurísticos de decisión (ver Wilson \& Dowlatabadi, 2007 para obtener más detalles). La mayor parte de estos modelos han surgido principalmente de la investigación realizada por economistas y en menor medida por psicólogos a pesar de que la toma de decisiones implica procesos cognitivos y emocionales (Kahneman, 2003; Kahneman \& Tversky, 1982; Stern, 2007). A este respecto, Stern (2007) hizo una revisión interesante relacionada con estudios sobre energía que implican la racionalidad limitada de la conducta del consumidor destacando su crítica acerca de la tendencia de estudiar dicha conducta desde la economía y no partiendo de otras ciencias conductuales no económicas. Asimismo, McKinsey y Company (2009) destaca que a pesar de los esfuerzos por fomentar comportamientos más racionales hay una mayor tendencia a estudiarlas desde la economía. Allí radica la importancia de evaluar la toma de decisiones bajo una perspectiva psicológica que permita, desde el planteamiento de los modelos descriptivos, conocer los procesos cognitivos y emocionales subyacentes al consumo responsable de energía. Por ello, este trabajo se inserta dentro de los modelos descriptivos de irracionalidad retomando el planteamiento de Kahenman y Tversky (1979) sobre la toma de decisiones, en este caso, relacionado con el consumo responsable de la energía eléctrica.

En el consumo responsable las personas toman decisiones cotidianamente sobre el uso de recursos (Chatterton, 2011; Jackson, 2008) dentro de su vivienda como los del agua, gas y energía eléctrica, entre otros. Dichas decisiones de consumo parecen estar caracterizadas por decisiones tanto complejas como sencillas (Barton et al., 2011). La toma de decisiones compleja implica un proceso mental lento y serial que Kahneman (2003) llama sistema razonado, y la toma de decisiones sencilla involucra un proceso mental rápido y sin esfuerzo identificado como sistema intuitivo. Asimismo, la toma de decisiones es un proceso dual en el que los mecanismos de ambos sistemas (razonado e intuitivo) operan en paralelo, en una sola ruta para procesar la información (Gilovich \& Griffin, 2002; Slogan, 2002) y pueden estar activos concurrentemente (Kahnemnan \& Frederick, 2002). Es decir, los dos sistemas intuitivo y razonado coexisten al momento en que la persona elije una opción y puede predominar alguno de estos. En el sistema intuitivo, las operaciones son rápidas, sin esfuerzo y cargadas de emociones controladas por hábitos y motivos, por lo tanto, son difíciles de modificar y controlar. En el 
sistema razonado, el proceso de operación es consciente y deliberado; es más lento, serial y demanda esfuerzo para seguir las reglas e instrucciones (Kahneman, 2003).

Adicionalmente, Stanovich y West (2002) plantean que estos dos sistemas difieren en que tienden a guiar diferentes tipos de tareas. El sistema intuitivo está altamente contextualizado, personalizado, socializado y asociado a conductas cotidianas. El sistema razonado sirve para descontextualizar y despersonalizar los problemas y su función es rechazar parte del medio que rodea la automatización del sistema intuitivo. De esta manera, podríamos decir que la toma de decisiones sobre el consumo de energía eléctrica parecería estar caracterizada por los dos sistemas ya que dicho consumo implica decisiones cotidianas con predominancia del sistema intuitivo como apagar o no la luz cada vez que se sale de un cuarto, y decisiones complejas donde destaca el sistema razonado como elegir entre usar un bombillo o foco ahorrador de luz blanca o cálida para una habitación.

Analizar la participación de estos dos sistemas en la toma de decisiones sobre el consumo de energía eléctrica requiere primero de investigación que permita explicar lo que hace la gente al consumir energía eléctrica, y segundo del desarrollo y prueba de instrumentos válidos y confiables. Sin embargo, aunque desde la psicología existen escasos estudios sobre la toma de decisiones en el consumo de energía eléctrica (Houston, 1983, Shen \& Saijo, 2009, Wijaya \& Tezuka, 2013), se hallaron algunas investigaciones en el consumo de otros servicios que ofrecen resultados interesantes tales como telefonía móvil (Chóliz, Villanueva \&Chóliz, 2009), aerolíneas (Prabhu \& Tellis, 2000), hoteles (Andrés, 2012) y seguro por daños (Kahneman, Schkade $\&$ Sunstein, 1998).

Respecto a los estudios sobre la toma de decisiones en el consumo de energía eléctrica, Houston (1983) realizó un estudio sobre la elección de un dispositivo de ahorro de energía en casa con 1081 propietarios de viviendas estadounidenses mediante el uso de un cuestionario enviado por correo en donde se incluía un problema con siete opciones de respuesta cerrada que iban desde pagar menos de 10 usd por año hasta más de 50 usd por año incluyendo la opción no sé o incierto. De esta manera, el autor encontró que 30\% de las personas elegían de manera irracional por falta de una regla de decisión que los guíe a comprender los datos; esto es, decidieron sin examinar costos y beneficios del dispositivo. Del mismo modo, el 70\% de los participantes realizó una evaluación analítica de la información al momento de elegir utilizando herramientas conceptuales para analizar el problema; esto significa que aplicaron reglas de decisión racional ante las diversas alternativas. Sin embargo, el estudio no especifica qué reglas de decisión fueron más efectivas. Por su parte, Shen y Saijo (2009) realizaron un estudio experimental en Shanghái sobre elección de refrigeradores y sistemas de aire acondicionado con base en la teoría de la utilidad. Participaron 1200 personas (600 vía Internet y 600 en entrevistas cara a cara), quienes respondieron un cuestionario en el que se presentaron seis atributos tanto para refrigerador (precio, rango de eficiencia energética, comparativo de consumo de energía respecto a otros modelos, consumo diario de energía, capacidad y reducción de ruido) como para aire acondicionado (precio, consumo por hora, espacio de enfriamiento, rango de eficiencia energética, comparativo de consumo de energía respecto a otros modelos). Su tarea consistió en elegir los atributos que tomarían en cuenta al comprar cada uno de estos equipos. Los resultados indican que las dos muestras el aire acondicionado con la función de purificación de aire y el refrigerador con reducción de ruido tuvieron mayor preferencia; asimismo, sólo los participantes entrevistados cara a cara optaron por el refrigerador con gran capacidad. Adicionalmente, Shen y Saijo (2009) observaron que los consumidores prefieren la información en las etiquetas de los aparatos al decidir su compra. Una ventaja del presente estudio es que incorpora herramientas de las nuevas tecnologías para realizar encuestas y una desventaja podría ser que los investigadores no especifican el tipo de estrategias que utilizaron los participantes para seleccionar los atributos importantes para comprar el aparato. Otra desventaja radica en el hecho que no pudieron verificar si los participantes en línea contaban con estos dos aparatos en casa.

Por otra parte, Wijaya y Tezuka (2013) compararon el reemplazamiento y compra de aparatos en dos ciudades de Indonesia. Participaron 100 amas de casa seleccionadas aleatoriamente y entrevistadas en su domicilio utilizando un cuestionario que enfatizaba dos temas: reemplazo de aparatos y toma de decisiones para la compra de aparatos nuevos (factores que influenciaban la compra y conocimiento del precio de electricidad) en una escala Likert con cinco opciones de respuesta (desde $1=$ ningún conocimiento hasta 5 = alto conocimiento). Los aparatos que más usaban 
fueron lavadoras, olla de arroz, lámparas, ventiladores, aire acondicionado, refrigeradores y televisores y bomba de agua. Asimismo, Wijaya y Tezuka (2013) encontraron que la información juega un papel importante ya que ayuda al comprador a tomar la decisión considerando características como precio, calidad, garantía, uso amigable, tecnología, seguridad, accesorios, tipo, marca y país de origen, destacando el precio y calidad. Respecto a los factores que influyen en la compra de aparatos, se tienen precio, calidad, seguridad, marca y amigabilidad. La falta de comprensión de los precios de electricidad podría ser la razón por la cual el consumo de energía no fuere seleccionado en ninguna de las dos ciudades como factor principal a la hora de tomar decisiones en la compra de los aparatos. Como limitaciones del presente estudio se identifica el hecho que el tamaño de la muestra es pequeño para incluir dos ciudades de un país. Por otra parte, aunque se reportan los atributos seleccionados por las participantes no se especifica la frecuencia con que tomarían en cuenta este tipo de atributos al comprarlos ni las estrategias utilizadas por las mismas para analizar los atributos al elegir. Parece que estos resultados pueden tener un sesgo por deseabilidad social de las participantes.

A continuación se exponen los estudios sobre toma de decisiones en otro tipo de servicios. En España se realizó un estudio sobre el servicio de telefonía móvil con 2486 estudiantes de secundaria y preparatoria utilizando una escala tipo likert para creencias sobre la imagen del móvil y funciones del móvil con tres dimensiones (afectivo-social, entretenimiento y comunicación) y encontraron que las mujeres prefieren usar el móvil como herramienta psicológica para hacer frente a diferentes estados de ánimo guiadas por la emoción (Chóliz et al., 2009). Por su parte, Prabhu y Tellis (2000) realizaron un estudio con 19 estudiantes universitarios sobre elección de un vuelo de los Ángeles a Londres por sus atributos (simulando por computadora los atributos (aerolínea, precio y calidad) y señalando al final de la elección sus claves o reglas para tomar decisiones. De esta forma, encontraron que los participantes tomaban decisiones basados en la información respecto al precio y en menor medida en la combinación de calidad y precio por el servicio que implica analizar las alternativas antes de elegir. Asimismo, hallaron que las reglas para tomar decisiones eran más analíticas y ponderaban los atributos antes de decidir. Aunque los resultados son interesantes, no se pueden generalizar dado el tamaño de la muestra empleada y el sesgo de escolaridad.
Por su parte, Andrés (2012) realizó un estudio sobre las preferencias de clientes virtuales respecto a la reservación en línea de una habitación de hotel en España con la finalidad de analizar los factores que influyen en el precio justo percibido. Para ello utilizó una simulación en línea elaborada ex profeso para el estudio con opciones de elección entre 5 hoteles, considerando el efecto moderado de la estrategia del precio y el tipo de estrategia de elección. Participaron 541 internautas quienes habían realizado al menos una reserva de hotel en línea. Primeramente, recibieron una invitación vía correo para participar; si aceptaban, recibían la clave de acceso a la simulación por la misma vía. La simulación contenía 5 hoteles con sus servicios, tarifas y fotografías. La tarea del participante era elegir uno haciendo la reservación en línea. Una vez hecha la reservación, el participante recibía vía correo electrónico un cuestionario para identificar los factores que influyen en el precio justo percibido y las consecuencias de los precios percibidos como justos/injustos. El cuestionario comprendía seis atributos (lealtad, satisfacción con el precio, confianza en la decisión, justicia percibida en el precio, familiaridad con reservas de hoteles en línea y precio de referencia). Los resultados indicaron una mayor preferencia por el precio al tomar decisiones cuando los precios son percibidos como justos, esto es, los consumidores están más satisfechos con el precio. Asimismo, Andrés (2012) halló que los consumidores usan un precio de referencia para determinar si el precio es justo o injusto. Cuando los usuarios están más familiarizados con la reserva vía internet, los consumidores tienden a percibir los precios más justos. Por otra parte, el autor encontró una clara relación entre la satisfacción por el precio pagado y la confianza en la decisión realizada. La ventaja del presente estudio es el uso de la simulación planteando la resolución de un problema o situación real.

En otro estudio, Kahneman, Schkade y Sunstein (1998) hicieron una investigación sobre elección de un seguro por daños, en Estados Unidos de América, en el cual participaron 899 jueces adultos. Los participantes respondieron a 10 viñetas en una escala de siete puntos (desde $0=$ ningún castigo hasta 6 = castigo extremadamente severo) sobre decisiones referentes al castigo económico para la empresa que causó dańos a la gente por usar un producto determinado. Kahneman, et al. (1998) obtuvieron resultados positivos hacia recompensar a la gente tomando decisiones razonadas. La ventaja del presente estudio es que utiliza vińetas que plantean situaciones en las que el participante se puede identificar con el personaje sin sentirse aludido. 
De estos estudios (Andrés, 2012; Chóliz et al., 2009; Houston, 1983; Kahneman et al., 1998; Prabhu \& Tellis, 2000; Shen \& Saijo, 2009, Wijaya \& Tezuka, 2013), se destaca que: (1) las personas pueden elegir tanto de manera intuitiva como razonada dependiendo del tipo de servicio que reciban; (2) la mayor parte de los estudios se basa en la elección de atributos físicos (capacidad y reducción de ruido del refrigerador, purificador de aire acondicionado), económicos (precio, calidad y recompensa), mercadológicos (marca, calidad, familiaridad, información el producto) y psicológicos (satisfacción, confianza, recompensa, seguridad); (3) en escasos estudios se midieron las estrategias utilizadas por los participantes al decidir, esto es, identificar qué sucede en el proceso de toma de decisiones; (4) la mayoría de las elecciones en el consumo de servicios parecen realizarse de manera más razonada que intuitiva y (5) la toma de decisiones puede ser medida a través de cuestionarios enviados por correo o en línea, cuestionarios en entrevistas personales, simulaciones por computadora y problemas ficticios o viñetas.

El uso de problemas ficticios o vińetas para medir la toma de decisiones respecto al uso de un dispositivo para ahorro de energía (Houston, 1983), reservar una habitación en un hotel (Andrés, 2012) y para utilizar un seguro por daños (Kahneman et al, 1998) permitieron medir las elecciones de los participantes. Los problemas ficticios o viñetas son pequeñas historias de situaciones a resolver que se les presentan por escrito a las personas; se les puede pedir a las personas que se pongan en el lugar del personaje y preguntarles qué harían o qué creen que haría el personaje (Mar, Oatley, Djikic \& Mullin, 2012; Oatley, 2011) de manera que no se sientan aludidos.

La técnica de las viñetas se apoya en los supuestos de que hay relación entre la simulación y la narrativa ficción (Bal \& Veltkamp, 2013; Oatley, 2011). La narrativa ficción es la manera de pensar acerca de lo que es posible y de lo que las personas con metas conciben como alternativas que deben enfrentar (Bruner, 1986). La ficción es un tipo de simulación que ofrece formas para enfrentar diferentes alternativas ante la toma de decisiones (Oatley, 2011) ya que son situaciones familiares que capturan la atención del lector. Dentro de la ficción se han destacado tres procesos psicológicos: identificación, empatía - atribuir emociones a los personajes - y memoria autobiográfica - recurrir a eventos previos (Mar \& Oatley, 2008; Oatley, 1999). De esta manera, al enfrentarse ante las posibles alternativas el lector de la vińeta recurre a alguno o varios de estos procesos psicológicos; adicionalmente, la toma de decisiones ante situaciones familiares lo alientan a responder debido a su corta extensión y originalidad (Mar et al., 2011). El uso de viñetas-situaciones narrativas en las cuales se presentan alternativas-en la medición de toma de decisiones ayudaría a expresar una complejidad de pensamientos, emociones y acciones en la investigación científica acerca de cogniciones y conductas (Mar et al., 2011; Reidl, Guillén, Sierra \& Joya, 2002). Por su parte, Tversky (2004) y colaboradores (Tversky, Heiser \& Morrison, 2013) plantean que, el 60\% del tiempo, las narraciones detallan algo de la vida de la persona, abstrayendo y comprimiendo información acerca de la misma. El espacio es estático en estas narraciones, pero se hace dinámico al describirlo o leerlo. Por ejemplo, al describir la tienda como lugar de compras, esta se convierte en un espacio dinámico donde se exhiben productos, venden, compran, pagan y dan cambio, entre otras cosas. Por lo anterior, las viñetas narrativas parecen ser una opción viable para medir la toma de decisiones sobre consumo de energía, porque mediante narraciones cortas parecen propiciar que el lector se enfrente a diferentes alternativas para elegir una, se identifique con el personaje sin sentirse aludido y abstraiga información de su vida antes de decidir.

Por lo anteriormente expuesto, la psicología puede jugar un papel importante en la evaluación de la conducta del consumidor ante los patrones de consumo responsable de energéticos dentro de la vivienda para reducir emisiones de gases contaminantes, beneficiar la economía familiar y conocer si los sistemas intuitivo y razonado participan en la toma de decisiones de dicho consumidor. La búsqueda de instrumentos válidos y confiables que permitan valorar el comportamiento de las personas hacia el consumo responsable llevó al desarrollo de uno en particular que permitiera evaluar el consumo de energía eléctrica en las viviendas (con reactivos tipo vińetas narrativas). Lo anterior se hace posible tomando como base el supuesto de que la toma de decisiones en el consumo de energía eléctrica puede ser medida mediante la elección en un continuo utilizando vińetas narrativas alusivas a la compra y uso de aparatos eléctricos domésticos. Es así como el propósito del presente estudio es precisamente documentar las características psicométricas de dicho instrumento; a este fin se realizó un estudio exploratorio de campo (Kerlinger \& Lee, 2002). 


\section{Método}

La variable toma de decisiones se define, de acuerdo con Kahneman y Tversky (1979), como la elección de una opción de entre varias alternativas. En el caso particular del consumo de energía eléctrica (EE) en la vivienda, la elección está determinada por dos sistemas: (a) intuitivo y (b) razonado.

Para llevar a cabo la validación del instrumento la investigación se estableció una división en: (1) elaboración del instrumento y validación de contenido (2) validez de constructo.

\section{Elaboración del instrumento y validación de contenido}

Objetivo. Obtener la validez de contenido de un instrumento que mida la toma de decisiones, razonada e intuitiva, sobre el consumo de energía eléctrica utilizando viñetas narrativas.

\section{Método}

Participantes. Participaron siete jueces expertos (cuatro mujeres y tres hombres) todos ellos psicólogos con estudios de doctorado e investigadores de la UNAM, dos de ellos especialistas en toma de decisiones. Asimismo, el instrumento se aplicó de manera individual a 69 mujeres voluntarias que supieran leer y escribir a quienes se les pidió su consentimiento informado (carta que firmaban en la primera hoja del cuestionario) para participar en el estudio. Se excluyeron aquellas mujeres cuya nacionalidad fuera extranjera o que tuvieran alguna alteración conductual visible. La edad promedio fue de 23 años $(D E=2.2)$. El $27 \%$ reportó tener estudios de primaria, $25 \%$ secundaria, $23 \%$ preparatoria y $25 \%$ licenciatura. La muestra fue de tipo no probabilística e intencional (Lohr, 2000).

Instrumento. Construcción. Para este instrumento se elaboraron 20 viñetas narrativas (ítems) sobre toma de decisiones relacionadas con el consumo de energía eléctrica, 10 viñetas relacionadas con toma de decisiones intuitiva (decisiones rápidas y cargadas de emociones) y 10 con toma de decisiones razonada (decisiones lentas, conscientes y deliberadas) retomando el concepto de toma de decisiones de Kahneman y Tversky (1979). Las vińetas diseñadas incluyeron los procesos psicológicos identificados por Mar y
Oatley (2008): identificación (todas eran mujeres), empatía (como emociones "el refrigerador que le encantó" o algo que no le gustaba) y memoria autobiográfica (tal como ver el televisor, compra de electrodomésticos, bombillos o focos para la casa). Para ello se describieron situaciones, mediante narraciones cortas, sobre compra y uso de aparatos eléctricos utilizados en el hogar en espacios como tiendas departamentales, habitaciones de la casa y supermercados (Tversky, 2004; Tversky et al, 2013). Adicionalmente, en el diseño de las viñetas se consideró que el consumo de energía eléctrica (CEE) parece estar caracterizado por decisiones tanto complejas como sencillas (Barton et al., 2013; Miirosa et al., 2011) por lo que se tomó esto en cuenta al momento de redactarlas.

Procedimiento. Como primera medida se concertó una cita previa con los jueces expertos a quienes se les presentaron 20 vińetas narrativas por escrito en tarjetas independientes, y en otras dos tarjetas las definiciones sobre toma de decisiones intuitiva (TDI) y toma de decisiones razonada (TDR). La tarea de los jueces consistió en clasificar las situaciones de acuerdo con las definiciones que se les presentaron. Debido a que los jueces expertos tenían estudios superiores a los de la población objetivo, pero su opinión era importante, se decidió evaluar el instrumento con mujeres quienes tuvieran las mismas características de las personas para las cuales se desarrolló el instrumento (Clark-Carter, 2002). Para ello se acudió a la vivienda de 69 mujeres y se les preguntó si tenían tiempo para contestar la encuesta sobre toma de decisiones. Si aceptaban se iniciaba la encuesta; si no se programaba una cita posterior. Se les presentaron las mismas 20 viñetas narrativas en una hoja y se les proporcionaron las definiciones sobre TDI y TDR en dos tarjetas; a continuación se les pidió que las tomaran en consideración al dar sus respuestas relacionando cada viñeta de acuerdo con las definiciones. La encuestadora anotaba las respuestas de las participantes. Al finalizar, se les daba las gracias y se les obsequiaba un folleto de ahorro de energía en la vivienda.

Análisis de datos. Para el índice de concordancia entre jueces expertos se utilizó la siguiente fórmula: total de acuerdos / (acuerdos + desacuerdos) x 100. Para el acuerdo interjueces (Clark-Carter, 2002) se calcularon frecuencias y porcentajes por viñeta esperando un índice de concordancia interjueces de $85 \%$. 


\section{Resultados}

Referente a los jueces expertos se obtuvo un $100 \%$ de concordancia entre jueces. En la tabla 1 se muestran los porcentajes de acuerdo interjueces; de las 20 viñetas narrativas elaboradas se observa que 6 vińetas tuvieron un promedio de acuerdo menor al $87 \%$, tomando como criterio que el $80 \%$ dijera que la viñeta sí estaba relacionada con la definición toma de decisiones intuitiva o razonada. A partir de estos resultados se elaboró el instrumento con las 14 viñetas rescatadas en esta primera selección, quedando así siete viñetas para TDI $(1,3,5,6,7,8$ y 10$)$ y siete viñetas para $\operatorname{TDR}(1,2,4,6,8,9$ y 10$)$.

En el diseño de las viñetas que conforman el instrumento TDCEE se consideró que el CEE parece estar caracterizado por decisiones tanto complejas como sencillas (Barton et al., 2013; Miirosa et al., 2011) por lo que se incluyeron en primera instancia reactivos de decisiones complejas y decisiones sencillas. Sin embargo, una vez realizado el análisis de datos quedaron más viñetas consideradas en las decisiones complejas.

Dentro de las viñetas diseñadas para el estudio se incluyeron los procesos psicológicos de identificación, empatía y memoria autobiográfica (Mar \& Oatley, 2008). De esta manera, las participantes tendrían opciones frente a diferentes alternativas para elegir una, identificándose con el personaje sin sentirse aludidas en situaciones que les fueren familiares (Mar, et al. 2011; Reidl, et al. 2002).

\section{Tabla 1}

Porcentaje de acuerdo interjueces para las viñetas $n=69$

\begin{tabular}{ccc}
\hline & \multicolumn{2}{c}{ Sí corresponde a toma de decisiones } \\
\hline Viñeta & Intuitiva & Razonada \\
\hline 1 & 87 & 88 \\
\hline 2 & 25 & 87 \\
\hline 3 & 93 & 42 \\
\hline 4 & 28 & 90 \\
\hline 5 & 93 & 39 \\
\hline 6 & 94 & 97 \\
\hline 7 & 87 & 35 \\
\hline 8 & 86 & 88 \\
\hline 9 & 41 & 91 \\
\hline 10 & 96 & 90 \\
\hline
\end{tabular}

Por otra parte, las participantes comprendían el texto ya que se incluyeron emociones y espacios (tienda, casa) que se volvían dinámicos al leerlos (Tversky, 2004; Tversky et al, 2013).

\section{Validez de constructo}

Objetivo. Determinar la validez de constructo del instrumento de toma de decisiones sobre el consumo de energía eléctrica.

\section{Método}

Participantes. La muestra fue de tipo no probabilística e intencional (Lohr, 2000). El instrumento lo respondieron voluntariamente 159 amas de casa residentes de una unidad habitacional al sur de la Ciudad de México quienes sabían leer y escribir. Se excluyeron aquellas amas de casa tuvieran alguna alteración conductual visible. Se seleccionaron amas de casa en razón de que son las principales consumidoras de bienes y servicios dentro del hogar (Nielsen Company, 2011). El ingreso familiar mensual fluctuó entre $\$ 3,000$ (229 USD; 162 euros) y $\$ 6,000$ (458 USD; 332 euros). El $16 \%$ de las participantes tenía estudios de primaria, 28\% de secundaria, $17 \%$ carrera técnica, $22 \%$ preparatoria y $17 \%$ de licenciatura. El rango de edad de las amas de casa entrevistadas fluctuó entre 18 y 80 años, con una media de 38 años $(D E=13.2)$.

Instrumento. Se elaboró un instrumento con las 14 vińetas denominado Toma de decisiones sobre consumo de energía eléctrica (TDCEE). Dichas viñetas fueron retomadas de la primera selección utilizando una escala con cinco opciones en un continuo que fue de nunca (0) hasta todo el tiempo (4). De esta manera las participantes al tener opciones de qué harían (desde nunca hasta todo el tiempo) se enfrentaban a diferentes alternativas para elegir una identificándose con el personaje sin sentirse aludidas en situaciones familiares (Mar, et al. 2011; Reidl, et al. 2002) tales como compra o uso de aparatos eléctricos, compra o uso de bombillos o focos, y uso de electrodomésticos. Se buscó que la confiabilidad Alpha de Cronbach fuera mayor a .60 .

Procedimiento. Se acudió a la vivienda del ama de casa y se le preguntó si tenía tiempo para contestar la encuesta sobre consumo de energía en la casa. Si el ama de casa aceptaba se le daba el cuestionario y una pluma 
para que contestara, si no se le preguntaba qué día y a qué hora podía contestar la encuesta. Si la persona decía que no le interesaba o que no podía se le daba las gracias y se tocaba en la siguiente vivienda. Las participantes leyeron cada viñeta y tacharon qué probabilidad habría de que hicieran lo mismo si estuvieran en el lugar de la protagonista. Al finalizar la encuesta se les agradeció su participación voluntaria y se les entregó un folleto sobre ahorro de energía eléctrica en la vivienda.

Análisis de datos. Con el fin de identificar algún agrupamiento en los reactivos aplicados, se realizó un análisis factorial exploratorio (Nunnally y Bernstein, 1995). Para lo cual se siguieron tres pasos: discriminación de reactivos, análisis factorial de reactivos con cargas factoriales iguales o superiores a .40 y estimación de consistencia interna de los factores por medio del alfa de Cronbach. Para la discriminación de reactivos se consideraron los resultados de la prueba $t$ al comparar los grupos con puntajes altos y bajos. En el análisis factorial se incluyeron los reactivos que discriminaron $(p<.05)$. Se obtuvo la estructura factorial mediante el método de componentes principales con rotación varimax. Para seleccionar los reactivos se consideró un valor propio (eigen) de uno o superior y una carga factorial mínima de .40 .

Resultados. La tabla 2 muestra las viñetas que discriminaron $(p<.05)$ al comparar las medias de los grupos con puntajes altos y bajos quedando ocho viñetas

\begin{tabular}{|c|c|c|c|c|c|c|c|c|}
\hline Reactivo/viñeta & grupo & $\mathrm{n}$ & media & DE & $\mathrm{F}$ & sig & $t$ & sig \\
\hline \multirow{2}{*}{1} & alto & 49 & 2.5 & 0.05 & 7.51 & .008 & -3.616 & .001 \\
\hline & bajo & 50 & 2.5 & 0.01 & & & & \\
\hline \multirow{2}{*}{2} & alto & 49 & 1.51 & 1.06 & 13.77 & .000 & -2.629 & 011 \\
\hline & bajo & 50 & 2.39 & 1.61 & & & & \\
\hline \multirow{2}{*}{3} & alto & 49 & 1.6 & 0.81 & 12.35 & . 001 & -2.74 & .008 \\
\hline & bajo & 50 & 2.36 & 1.38 & & & & \\
\hline \multirow{2}{*}{4} & alto & 49 & 2.17 & 1.27 & 1.32 & .255 & -2.074 & .003 \\
\hline & bajo & 50 & 3.21 & 0.05 & & & & \\
\hline \multirow{2}{*}{5} & alto & 49 & 2.71 & 1.4 & 0.162 & 688 & -2.78 & .007 \\
\hline & bajo & 50 & 3.5 & 0.04 & & & & \\
\hline \multirow{2}{*}{6} & alto & 49 & 3.7 & 0.03 & 9.92 & . 002 & 1.993 & .055 \\
\hline & bajo & 50 & 3.18 & 0.05 & & & & \\
\hline \multirow{2}{*}{7} & alto & 49 & 3.31 & 0.43 & 7.42 & .008 & -1.332 & 187 \\
\hline & bajo & 50 & 3.7 & 0.03 & & & & \\
\hline \multirow{2}{*}{8} & alto & 49 & 2.51 & 1.44 & 1.234 & .271 & -2.342 & .001 \\
\hline & bajo & 50 & 3.64 & 0.03 & & & & \\
\hline \multirow{2}{*}{9} & alto & 49 & 2.29 & 1.4 & 0.874 & .353 & -0.531 & .594 \\
\hline & bajo & 50 & 3.09 & 0.18 & & & & \\
\hline \multirow{2}{*}{10} & alto & 49 & 2.31 & 1.4 & 0.665 & .421 & -0.2442 & .017 \\
\hline & bajo & 50 & 2.79 & 1.08 & & & & \\
\hline \multirow{2}{*}{11} & alto & 49 & 2.6 & 1.11 & 5.448 & .023 & -2.814 & .192 \\
\hline & bajo & 50 & 3.33 & 0.66 & & & & \\
\hline \multirow{2}{*}{12} & alto & 49 & 3.94 & 0.02 & 0.963 & .330 & -2.859 & .482 \\
\hline & bajo & 50 & 3.12 & 0.35 & & & & \\
\hline \multirow{2}{*}{13} & alto & 49 & 1.17 & 0.747 & 0.053 & 819.000 & -0.538 & .593 \\
\hline & bajo & 50 & 1.36 & 0.856 & & & & \\
\hline 14 & alto & 49 & 1.83 & 1.09 & 3.626 & .061 & -2.952 & .004 \\
\hline
\end{tabular}


$(1,2,3,4,5,8,10$ y 14$)$ que posteriormente se incluyeron en el análisis factorial.

Como se observa en la tabla 3, los resultados de análisis factorial evidenciaron dos factores toma de decisiones intuitiva - TDI -y toma de decisiones razonada- TDR con cargas entre .65 y .78 y una confiabilidad alta $(a=.80$ y a $=.77$, respectivamente). Cada factor quedó conformado por tres reactivos respectivamente. El análisis factorial de componentes principales arrojó una estructura de dos factores en el que las dimensiones atribuidas a toma de decisiones obtuvieron cargas factoriales superiores a .40 explicándose el $32.5 \%$ de la varianza. Los resultados factoriales que se obtuvieron para el resto de los ítems no alcanzaron pesos factoriales de suficiente importancia en ninguno de los dos factores que se buscaban.

\section{Tabla 3}

Validez factorial de constructo de las viñetas de toma de decisiones sobre consumo de energía eléctrica.

\begin{tabular}{lcc}
\hline \multicolumn{2}{c}{ Factores } \\
\multicolumn{1}{c}{ Voma de decisiones } \\
\hline 10. escuchar ruido & $\begin{array}{c}\text { Intuitiva } \\
\alpha=.77\end{array}$ & $\begin{array}{c}\text { Razonada } \\
\alpha=.80\end{array}$ \\
\hline 5. ver iluminado & .78 & \\
\hline 3. calor de hogar & .77 & \\
\hline 14. misma marca & .73 & .68 \\
\hline 1. precio & & .67 \\
\hline 2. más información & & .65 \\
\hline
\end{tabular}

Se observó que la mayoría de las viñetas que se incluyeron en el análisis factorial contenían dos o más atributos (marca, precio y consumo en watts; precio y vida útil), lo que indica que pueden ser decisiones más complejas.

Se encontraron dos dimensiones TDI (sencillas) y TDR (complejas) claramente definidas. Lo anterior indica que con este instrumento se puede medir la TD por separado aunque sea un proceso paralelo. El instrumento final se muestra en el Apéndice.

\section{Discusión}

Como se mencionó anteriormente, el objetivo del presente estudio fue documentar las características psicométricas de un instrumento, con el fin de evaluar la toma de decisiones sobre el consumo de energía eléctrica TDCEE empleando vińetas narrativas y partiendo de que la toma de decisiones es un proceso dual razonado e intuitivo (Kahneman, 2003; Kahneman \& Tversky, 1979). Los resultados muestran que el instrumento TDCEE cumple con criterios psicométricos aceptables.

Con respecto a la escala TDCEE se observaron dos dimensiones claramente definidas en la toma de decisiones tanto intuitiva como razonada; esto apoya el planteamiento de Kahneman y Tversky (1979) y Kahneman (2003), respecto a que estas dos formas de toma de decisiones están inmersas en un proceso dual pero diferenciadas por la manera en que se elige, bien sea esta lenta o rápida.

De esta manera se define la toma de decisiones razonada como la elección de una opción de consumo de energía eléctrica (CEE) de entre varias alternativas mediante un proceso lento y serial. $Y$ la toma de decisiones intuitiva como la elección de una opción de CEE entre varias alternativas de manera rápida y cargada de emociones. De esta manera los sistemas intuitivo y razonado difieren en que tienden a guiar diferentes tipos de tareas (Stanovich \& West, 2002) y decisiones (Barton et al., 2013; Mirosa et al., 2011).

Los índices de validez estándar demostraron que esta escala es sensible y capaz de distinguir entre decisiones razonadas e intuitivas en el CEE. Esto cobra relevancia porque al medir la TD en contextos de energía doméstica será necesario incluir ambas. Lo anterior concuerda con los resultados obtenidos en el estudio de elección de un dispositivo de ahorro energía eléctrica en la vivienda (Houston, 1993) en donde los participantes eran propensos a decidir tanto de manera analítica como emocional.

El instrumento generado en la presente investigación proporciona riqueza empírica y validez ecológica al planteamiento de toma de decisiones de Kahneman (2003). No obstante, sería recomendable continuar la validez ecológica del mismo aplicándolo en otros contextos y con diferentes participantes.

La inclusión de componentes del proceso psicológico de la narración en el diseño de las viñetas del presente estudio tales como identificación, empatía y memoria autobiográfica (Mar \& Oatley, 2008) y espacio y el tiempo (Tversky et al., 2013) permiten crear situaciones que invitan al tomador 
de decisiones en contextos domésticos a experimentar emociones de identificación -recordadas o novedosas- a través del espacio - tiempo narrativo. Lo anterior constituye una guía apropiada para los investigadores interesados en la medición psicológica.

Los hallazgos del presente estudio indican que es factible que la TD sea medida mediante viñetas o situaciones ficticias. Lo anterior concuerda con estudios de Houston (1993) y Kahneman et al. (1998) quienes midieron las elecciones por medio de viñetas o problemas ficticios.

En congruencia con estudios previos sobre toma de decisiones en consumo de servicios (Houston, 1993; Kahneman et al., 1998) en el presente estudio se encontró que el uso de viñetas para la medición psicológica de toma de decisiones parece ser una estrategia de recolección de datos eficaz. Lo anterior sugiere una reducción de sesgo en las respuestas debido a deseabilidad social (Crowne \& Marlowe, 1964; Domínguez, 2008; Pérez, Labiano \& Brusasca, 2010), dado que permite obtener información sin que el entrevistado se sienta aludido (Mar et al., 2011; Oatley, 2011).

Respecto al número de viñetas que conforman el instrumento final se encontró que para las dos dimensiones de TD se incluyeron más viñetas asociadas a la toma de decisiones compleja. Lo que contrasta con el planteamiento de Gilovich y Griffin (2002) y Slogan (2002) sobre que la toma de decisiones es un proceso dual que opera en paralelo.

En el presente estudio se halló que la mayor parte de las viñetas que comprenden las dos dimensiones encontradas (TDI - TDR) contienen dos o más atributos (precio-vida útil, precio, marca y consumo en watts), lo que concuerda con los hallazgos de estudios previos sobre la preferencia al decidir tomando en cuenta dos o más atributos como precio y familiaridad en reservas de hotel (Andrés, 2012), reducción de ruido y gran capacidad en refrigeradores (Shein \& Saijo, 2009) y precio, seguridad, marca y amigabilidad de los aparatos eléctricos (Wijaya \& Tezuca, 2013).

Este trabajo refleja que la TD en el CEE puede ser medida desde la perspectiva de los modelos descriptivos en la cual se enmarca el modelo de TD de Kahneman (2003) y Kahneman y Tversky (1979). En concordancia con Stern (2007), lo anterior permite vislumbrar que se puede estudiar la TD no sólo desde la economía (McKinsey \& Company,
2009) sino también desde las ciencias conductuales no económicas como la psicología.

Por otra parte, este estudio aporta un instrumento que podría utilizarse para conocer la toma de decisiones respecto al CEE en conjunto con la medición de otras variables psicológicas (Mirosa et al., 2011) y en un futuro contribuir con los resultados para el desarrollo de una estrategia de intervención ambiental especifica que involucre a los ciudadanos en el consumo responsable en el marco del pacto de la Ciudad de México (Natyelly, 2010). Cabe destacar que aunque para las amas de casa del presente estudio el CEE representó un gasto del 18\% del gasto familiar (cifra menor con la reportada por el INEGI, 2012) su CEE es un contaminante silencioso debido a que el suministro de energía eléctrica para la Ciudad de México proviene de plantas termoeléctricas (CFE, 2010) que emiten gases contaminantes al aire (Comisión Ambiental Metropolitana, 2010; PNUMA, 2013).

Este estudio contribuye, desde la psicología, al desarrollo de instrumentos sobre el consumo de energía eléctrica. Para estudios futuros queda el indagar si las respuestas al instrumento TDCEE se asocian al consumo de energía eléctrica en la vivienda. Asimismo, es necesario desarrollar instrumentos que midan los procesos cognitivos y emocionales tales como memoria, percepción, motivación para la toma de decisiones en el consumo responsable.

Una posible limitación del presente estudio es que el instrumento está diseñado y validado solamente con mujeres adultas de la Ciudad de México. No obstante, puede ser un referente de lo que sucede en los Estados de la República Mexicana debido a que gran parte de la población en la Ciudad de México proviene de diversos Estados (Instituto Nacional de Estadística, Geografía e Informática, 2010). De cualquier forma, para futuros trabajos, convendría aplicar el instrumento en el interior del país para conocer los resultados.

Es factible asumir que el instrumento construido y probado empíricamente para medir toma de decisiones -TDCEE- cumple con características psicométricas aceptables. Dicho instrumento representa una opción en la medición de variables psicológicas vinculadas con el consumo de energía eléctrica y es un primer paso en la búsqueda de procesos psicológicos que subyacen a dicho consumo en la vivienda de la Ciudad de México. 
Los resultados del presente estudio proporcionan información suficiente acerca de la validez de constructo de instrumento TDCEE para evaluar la toma de decisiones intuitiva y razonada. Sin embargo, valdría la pena comprobar estos hallazgos con una muestra de mayores proporciones. Finalmente, se puede afirmar que el instrumento TDCEE es una alternativa adecuada para la evaluación de la toma de decisiones con amas de casa.

\section{Referencias}

Andrés, E. (2012). Percepción de la justicia de precios por el consumidor en entornos virtuales ante diferentes estrategias de gestión de demanda. Tesis doctoral Universidad de Castilla-La Mancha España. Recuperado de https://ruidera.uclm.es/ xmlui/bitstream/handle/10578/3017/TESIS\%20 Andres\%20Mart\%C3\%ADnez.pdf?sequence=1

Bal, P., \& Veltkamp M (2013) How does fiction reading influence empathy? An experimental investigation on the role of emotional transportation. PLoS ONE 8(1). doi:10.1371/journal.pone.0055341

Barton, B., Blackwell, S., Carrington, G., Ford, R., Lawson, R., Stephenson, J., Thorsnes, P., \& Williams, J. (2013). Energy cultures: implication for policymakers. Centre for Sustainability, University of Otago: New Zealand.

Brohmann, B., Heinzle, S., Nentwich, J., Offenberger, U., Rennings, K., Schleich, J., \& Wüstenhagen, R. (2009). Sustainable energy consumption and individual decisions of consumers - review of the literature and research needs. Germany: Darmstadt, Freiburg, Karlsruhe, Mannheim, St. Gallen

Bruner, J. (1986). Actual minds, possible worlds. Cambridge, MA: Harvard University Press.

Chatterton, T. (2011). An introduction of thinking about energy behavior. A mult-model approach. Deparment of energy and climate change. London, UK.

Chóliz, M., Villanueva, V., \& Chóliz, M. (2009). Ellas, ellos y su móvil: Uso, abuso (¿y dependencia?) del teléfono móvil en la adolescencia. Revista Española de Drogodependencias, 34(1), 74-86.

Clark-Carter, D. (2002). Investigación cuantitativa en psicología. México: Oxford.

Comisión Ambiental Metropolitana (2010). Agenda de sustentabilidad ambiental para la zona metropolitana del Valle de México. México: Comisión ambiental metropolitana. Recuperado de http://biblioteca.semarnat.gob.mx/janium/ Documentos/Ciga/libros2009/CD000964.pdf

Comisión Federal de Electricidad (2010). Generación. Recuperado dehttp://www.cfe.gob.mx/QuienesSomos/ estadisticas/Paginas/Indicadoresdegeneración.aspx

Crowne, P., \& Marlowe, O. (1964). The approval motive: Studies in evaluative dependence. New York: Wiley.

Domínguez, A. (2008). Validez concurrente de la escala de deseabilidad social de Domínguez utilizando la escala de deseabilidad social de Marlowe-Crowne. Revista Iberoamericana de Diagnóstico y Evaluación Psicológica, 25(1), 125-139.

Gilovich, T. \& Griffin, D. (2002). Introduction heuristics and biases: Then and now. En T. Gilovich, D. Griffin $\&$ D. Kahneman (Eds.), Heuristics and biases. The psychology of intuitive judgment (pp.1-18). Cambridge University Press.

Heinzle, S. (2010) Behavioural models of decision making and implications for green marketing. Germany: St. Gallen

Houston, D. (1983). Implicit discount rates and the purchase of untried, energy-saving durable goods. The Journal of Consumer Research, 10(2) 236-246.

Instituto Nacional de Estadística, Geografía e Informática INEGI (2010). Censo de población y vivienda 2010. Resultados preliminares. Recuperado de http://www. censo2010.org. $\mathrm{mx} /$

Instituto Nacional de Estadística, Geografía e Informática INEGI (2012). Encuesta nacional de ingresos y gastos de los hogares (enigh) 2012. Principales resultados. Recuperado de http://www.inegi.org. $\mathrm{mx} /$ est/contenidos/Proyectos/Encuestas/Hogares/ regulares/Enigh/Enigh2012/tradicional/default.aspx

Jackson, T. (2008). Live better by consuming less?: Is There a "Double Dividend" in sustainable consumption? Journal of Industrial Ecology, 9 (1-2), 19-36. doi: 10.1162/1088198054084734

Kahneman, D. (2003). Maps of bounded rationality: Psychology for behavioral economics. The American Economic Review, 93(5), 1449-1475. doi: $10.1257 / 000282803322655392$

Kahneman, D., \& Frederick, S. (2002). Representativeness revisited: Attribute substitution in intuitive judgment. En T. Gilovich, D. Griffin \& D. Kahneman. (Eds.), 
Heuristics and biases. The psychology of intuitive judgment (pp.49-81). Cambridge University Press.

Kahneman, D., \& Tversky, A. (1979). Prospect theory: An analysis of decision under risk. Econometrica, 47(2), 263-291. Recuperado de: http://www.jstor. org/stable/1914185

Kahneman, D., Schkade, D., \& Sunstein, C. (1998). Shared outrage and erratic awards: The psychology of punitive damages. Journal of Risk and Uncertainty, 16, 49-86. doi: 10.1023/A:1007710408413

Kahneman, D., \& Tversky, A. (1982). On the study of statistical intuitions. Cognition, 11, 123-141. doi: http://dx.doi.org/10.1016/0010-0277(82)90022-1

Kerlinger, F., \& Lee, H. (2002). Investigación del comportamiento. Métodos de investigación en ciencias sociales ( $3^{a}$ ed.). México: Mc Graw Hill.

Lohr, S. (2000). Muestreo: Diseño y análisis. México: International Thompson.

Mar, R., \& Oatley, K. (2008). The function of fiction is the abstraction and simulation of social experience. Perspectives on Psychological Science, 3(3), 173-192. doi: 10.1111/j.1745-6924.2008.00073.x

Mar, R., Oatley, K., Djikic M., \& Mullin,J. (2011). Emotion and narrative fiction: Interactive influences before, during, and after reading. Cognition \& Emotion, 25(5), 818-833 doi: 10.1080/02699931.2010.515151

McKinsey \& Company, (2009). Pathways to a lowcarbon economy: Version 2 of the global greenhouse gas abatement cost curve. Recuperado de www. mckinsey.com/ clientservice/ccsi/pathways_low_ carbon_economy.asp

Mirosa, M., Lawson, R., \& Gnoth, D. (2011). Linking Personal Values to Energy-Efficient Behaviors in the Home Environment and Behavior 45(4), 455-475 doi: $10.1177 / 0013916511432332$

Natyelly, B. (2010). Pacto de la Ciudad de México: Diez acciones contra el cambio climático. Milenio. Recuperado de http://www.milenio.com/ print $/ 583155$

Nielsen Company (2011). Cambios en el mercado mexicano 2011. Recuperado de http://ftp.vaexposiciones.com/ descargas/cambiosdelmercadomexicano_nielsen.pdf

Nunnally, J. \& Berstein, I. (1995). Teoría psicométrica. México: Mc Graw Hill.
Oatley, K. (1999). Why fiction may be twice as true as fact: fiction as cognitive and emotional simulation. Review of General Psychology, 3(2), 101-117.

Oatley, K. (2011). In the minds of others. Scientific American Mind, 22(5), 62-67 doi: 10.1038/ scientificamericanmind1111-62

Organization for Economic Co-operation and Development / International Energy Agency (2005). Saving electricity in a hurry. París, Francia: IEA.

Pérez, M., Labiano, M., \& Brusasca, C. (2010). Escala de deseabilidad social: análisis psicométrico en muestra argentina. Evaluar, 10, 53-67.

Prabhu, J., \& Tellis, G. (2000). Do consumers even learn? Analysis of segment behavior in experimental markets. Journal of Behavioral Decision Making, 13, 19-34.

Programa de las Naciones Unidas para el Medio Ambiente PNUMA (2013). Consumo sostenible. Recuperado de http://www.unep.org/spanish/wed/ sustainableconsumption/

Reidl, L., Guillén, R., Sierra, G., \& Joya, L. (2002). Medición alternativa de las emociones de celos y envidia. México: Facultad de Psicología, Facultad de Estudios Superiores Zaragoza, UNAM.

Shen, J., \& Saijo, T, (2009). Does an energy effcency label alter consumers' purchasing decisions? A latent class approach based on a stated choice experiment in Sahngai. Journal of Environmental Management, 90, 3561-3573 doi: 10.1016/j.jenvman.2009.06.010

Shogren, J. (2012). Behavioural Economics and Environmental Incentives, OECD Environment Working Papers, No. 49, OECD Publishing. doi: http://dx.doi.org/10.1787/5k8zwbhqs1xn-en

Slogan, S. (2002). Two systems of reasoning. En T. Gilovich, D. Griffin \& D. Kahneman (Eds.), Heuristics and biases. The psychology of intuitive judgment (pp.379396). Cambridge University Press.

Stanovich, K., \& West, R. (2002). Individual differences in reasoning: implications for the rationality debate? En T. Gilovich, D. Griffin \& D. Kahneman. Heuristics and biases. The psychology of intuitive judgment (pp.421-440). Cambridge University Press.

Stern, N. H. (2007). The economics of climate change: The Stern Review. Cambridge,UK: Cambridge University Press. 
Tversky, B. (2004). Narratives of space, time, and life. Mind and Language, 19(4), 380-392. Recuperado de http://psych.stanford.edu/ -bt/memory/papers/03_ mila003.pdf

Tversky, B., Heiser, J., \& Morrison, J. (2013) Space, time and story In: B. Ross. (Ed.), Psychology of Learning and Motivation, 58 (pp.47-76). Elsevier.

Vandenberg, M., Carrico, A. \& Shultz, L. (2011). Regulation in the Behavioral Era. Minnesota Law Review 95, 715-781. doi
Wijaya, M., \& Tezuca, T. (2013). Measures for improving the adoption of higher efficiency appliances in Indonesian households: An analysis of lifetime use and decision-making in the purchase of electrical appliances. Applied Energy, 112, 981-987.doi: 10.1016/j.apenergy.2013.02.036

Wilson, C., \& Dowlatabadi, H. (2007). Models of decision making and residential energy use. Annual Review of Environment and Resources, 32, 169-203. doi: 10.1146/annurev.energy.32.053006.141137 


\section{Apéndice}

\section{Instrumento TDCEE}

Toma de decisiones intuitiva:

Daniela llega a su casa y enciende el televisor. A ella no le gustan los programas de televisión pero le gusta escuchar ruido.

En el lugar de Daniela usted haría lo mismo

- Todo el tiempo

- La mayor parte del tiempo

- A veces

- Casi nunca

- Nunca

Paola quiere comprar una lámpara nueva para su casa. Llega a la tienda y empieza a ver los diferentes modelos, pero no le gusta ninguno por lo que va a otra tienda en donde encuentra tres modelos que le gustan mucho y cuestan lo mismo. Decide comprar la lámpara que tiene más bombillos o focos porque le gusta ver iluminada su casa cuando es de noche.

En el lugar de Paola usted haría lo mismo

- Todo el tiempo

- La mayor parte del tiempo

- A veces

- Casi nunca

- Nunca
Karla llega a su casa mojada por la lluvia, mientras se seca y se cambia enciende el calentador eléctrico para sentir calor de hogar aunque en su departamento no se sienta frío.

En el lugar de Karla usted haría lo mismo

- Todo el tiempo

- La mayor parte del tiempo

- A veces

- Casi nunca

- Nunca

Toma de decisiones razonada:

Alejandra necesita cambiar el refrigerador de su casa. Llega a la tienda hay varios refrigeradores pero hay uno que le encantó, se acerca a verlo, lo abre y lo cierra y observa que tiene la misma marca que su lavadora actual, la cual le ha salido muy buena, por lo que decide comprarlo.

En el lugar de Alejandra usted haría lo mismo

- Todo el tiempo

- La mayor parte del tiempo

- A veces

- Casi nunca

- Nunca 